\title{
MASTER
}

\section{Particle Size Characteristics of Pyrotechnic PoWders}

A. A. Duncan

DEVELOPMENT DIVISION

DECEMBER 1976

(P.O. NO. 03-2530)

For

Sandia Laboratories

Albuquerque, New Mexico

\section{.}

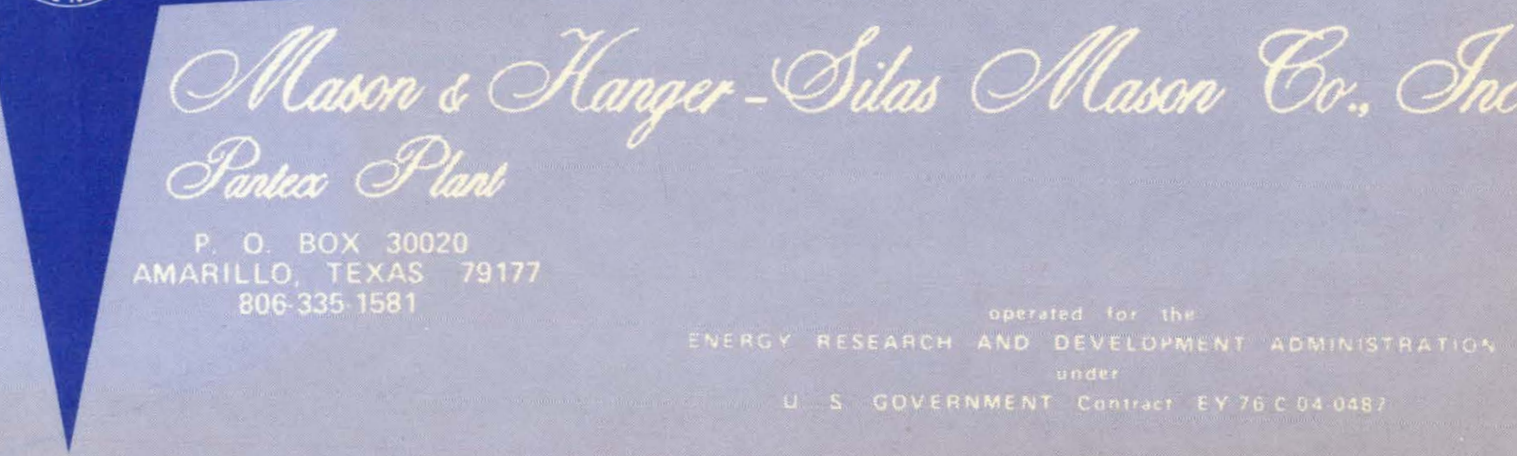




\section{DISCLAIMER}

This report was prepared as an account of work sponsored by an agency of the United States Government. Neither the United States Government nor any agency Thereof, nor any of their employees, makes any warranty, express or implied, or assumes any legal liability or responsibility for the accuracy, completeness, or usefulness of any information, apparatus, product, or process disclosed, or represents that its use would not infringe privately owned rights. Reference herein to any specific commercial product, process, or service by trade name, trademark, manufacturer, or otherwise does not necessarily constitute or imply its endorsement, recommendation, or favoring by the United States Government or any agency thereof. The views and opinions of authors expressed herein do not necessarily state or reflect those of the United States Government or any agency thereof. 


\section{DISCLAIMER}

Portions of this document may be illegible in electronic image products. Images are produced from the best available original document. 


\section{NOTICE}

This report was prepared as an account of work sponsored by the United States Government. Neither the United States nor the United States Energy Research and Development Administration, nor their employees, nor any of their contractors, subcontractors, or their employees, makes any warranty, express or implied, or assumes any legal liability or responsibility for the accuracy, completeness or usefulness of any information, apparatus, product or process disclosed, or represents that its use would not infringe privately-owned rights. 


\title{
Particle Size Characteristics of Pyrotechnic Powders
}

\author{
A. A. Durican \\ DEVELOPMENT DIVISION
}

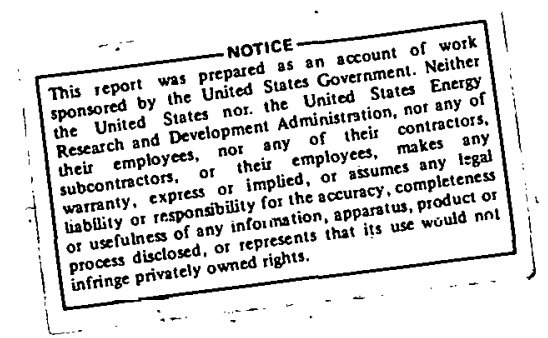

December 1976

(P.O. No. 03-2530)

For
Sandia Laboratories
Albuquerque, New Mexico 
Pyrotechnic powders supplied by Al Heckes of Sandia Laboratories, Albuquerque, have been characterized using a semi-automatic microscopy technique (Zeiss Analysis) to measure particle size distribution and specific surface area by both gas adsorption (static BET apparatus) and permeametry (Fisher Sub Sieve Sizer). Photomicrographs, specific surface area and mean particle sizes are given in this report.

\section{DISCUSSION}

Pyrotechnic powders supplied by Al Heckes (Sandia) were characterized by a microscopy technique using a semi-automatic measuring device (Zeiss Analyzer TGZ-3). This technique is capable of measuring those dimensions applicable to the shape of individual particles from photomicrographs. The usefulness of many microscopy techniques is limited due to single statistical values and the inability to distinguish individual particles when agglomerated. The Pantex technique is based on the measurement of individual particles where shape decides those dimensions to be measured (e.g. if ellipsoid the major and minor axis are measured). From dimensional measurements physical parameters can be calculated for individual particles so distribution and bulk powder characteristics can be generated.

In this study, physical parameters distribution for length, width, length/ width ratio, cross-sectional area, surface area, volume, equivalent circular diameter, equivalent spherical diameter, degree of sphericity and powder uniformity were calculated. From each parameter, distribution arithmetic mean values based on frequency and weight are given in Table I. Complete distribution values from which the mean values were calculated have been transmitted to Al Heckes.

Specific surface area determinations in this study were performed by gas adsorption and permeametry techniques. Gas adsorption isotherms were obtained using nitrogen as an absorbent at liquid nitrogen temperature $(\sim 76 \mathrm{~K})$. A static system, the Micrometrics Orr Surface-Area/PoreVolume Analyzer Model 2100D, was used for this analysis. Repeatability of this measurement is $0.02 \mathrm{~m}^{2} / \mathrm{g}$.

Permeametry surface area and average particle diameter were measured by the Fisher Sub-Sieve Sizer Apparatus. A series of average particle diameters are measured for each batch of material at various porosities so the proper air-flow to particle ratio may be observed. Achievement of this relationship is important so an agglomerated particle or crushed particle specific surface area is not reported. A plateau where the same average particle diameter for repeated porosity is obtained indicates proper air-flow around individual particles. Repeatability of this measurement is $\pm 0.005 \mathrm{~m}^{2} / \mathrm{g}$ and $0.2 \mathrm{um}$ for average particle diameter.

Results for each batch distribution mean, standard deviation, and specific surface area are found in Table I. 
FUTURE WORK

This concludes the work that was requested for these samples. 
Table.I. Zeiss Distribution Mean and Standard Deviation

Frequency

Weight

Sample Identification: Paliadium INCO 400, Lot PC 807

Length $(\mu \mathrm{m})$

Length Std. Dev.

Width $(\mu \mathrm{m})$

Width Std. Dev.

Length/Width Ratio

Cross-Sectional Area $\left(\mu \mathrm{m}^{2}\right)$

Surface/Particle $\left(\mu \mathrm{m}^{2}\right)$

Volume $\left(\mu \mathrm{m}^{3}\right)$

Equivalent Circular Diameter ( $\mu \mathrm{m})$

Equilvalent Spherical Diameter $(\mu \mathrm{m})$

Degree of Sphericity

Powder Uniformity

Zeiss Specific Surface Area $\left(\mathrm{m}^{2} / \mathrm{g}\right)$

BET Specific Surface $\left(\mathrm{m}^{2} / \mathrm{g}\right)$
2.86
1.41
1.62
1.83
1.76
4.55
19.60
8.07
2.15
2.00
0.84
4.78
2.025
1.415
4.89
0.71
2.65
0.84
1.86
13.46
60.52
35.19
3.89
3.63
0.80
5.79

Sample Identification: Palladium Englehard PD-325 Mesh

Length $(\mu \mathrm{m})$

Length Std. Dev.

Width $(\mu \mathrm{m})$

Width Std. Dev.

Length/Width Ratio

Cross-Sectional Area $\left(\mu \mathrm{m}^{2}\right)$

Surface/Particle $\left(\mu \mathrm{m}^{2}\right)$

Volume $\left(\mu \mathrm{m}^{3}\right)$

Equivalent Circular Diameter $(\mu \mathrm{m})$

Equivalent Spherical Diameter $(\mu \mathrm{m})$

Degree of Sphericity.

Powder Uniformity

Zeiss Specific Surface Area $\left(\mathrm{m}^{2} / \mathrm{g}\right)$

BET Specific Surface $\left(\mathrm{m}^{2} / \mathrm{g}\right)$
2.18

1.43

1.68

0.65

1.24

3.80

15.49

7.36

1.94

1.88

0.96

5.96 .

0.175

0.185

Sample Identification: Titanium Powder Alpha Product No. 99612 Lot 08057

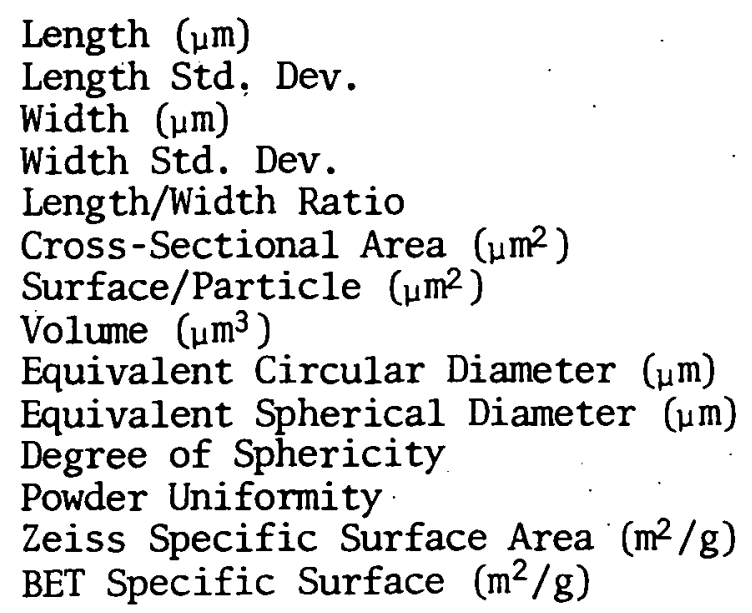

6.07

4.03

3.42

1.74

1.67

26.40

115.05

120.34

4.89

4.57

0.87

3.98 
Table I. Zeiss Distribution Mean and Standard Deviation (Cont'd)

Frequency Weight

Sample Identification: Boron Callery 2903-72-3 CCC 951299

Length $(\mu \mathrm{m})$

Length Std. Dev.

Width $(\mu \mathrm{m})$

Width Std. Dev.

Length/Width Ratio

Cross-Sectional Area $\left(\mu \mathrm{m}^{2}\right)$

Surface/Particle $\left(\mu \mathrm{m}^{2}\right)$

Volume $\left(\mu \mathrm{m}^{3}\right)$

Equivalent Circular Diameter ( $\mu \mathrm{m})$

Equivalent Spherical Diameter ( $\mu \mathrm{m})$

Degree of Sphericity

Powder Uniformity

Zeiss Specific Surface Area $\left(\mathrm{m}^{2} / \mathrm{g}\right)$

BET Specific Surface $\left(\mathrm{m}^{2} / \mathrm{g}\right)$
1.13

0.59

0.73

0.26

1.51

0.80

3.42

0.60

0.91

0.86

0.90

6.31

24.530

30.520
2.00

0.86

1.14

0.43

1.77

2.49

11.39

3.10

1.64

0.90

0.81

5.41

Sanple Identification: Aluminum -325 Mesh Job No. 5647 MRC-Mare Part No. 586700X01

Length $(\mu \mathrm{m})$

Length Std. Dev.

Width $(\mu \mathrm{m})$

Width Std. Dev.

Length/Width Ratio

Cross-Sectional Area $\left(\mathrm{um}^{2}\right)$

Surface/Particle $\left(\mu \mathrm{m}^{2}\right)$

Volume $\left(\mu \mathrm{m}^{3}\right)$

Equilvalent Circular Diameter ( $\mu \mathrm{m})$

Equilvalent Spherical Diameter ( $\mu \mathrm{m}$ )

Degree of Sphericity

Powder Uniformity

Zeiss Specific Surface Area $\left(\mathrm{m}^{2} / \mathrm{g}\right)$

BET Specific Surface $\left(\mathrm{m}^{2} / \mathrm{g}\right)$

$\begin{array}{rr}18.29 & 34.49 \\ 10.54 & 14.25 \\ 9.69 & 16.98 \\ 3.86 & 7.30 \\ 1.84 & 2.10 \\ 172.58 & 626.19 \\ 686.02 & 2580.01 \\ 1685.73 & 10344.39 \\ 13.20 & 25.88 \\ 11.88 & 22.98 \\ 0.86 & 0.79 \\ 5.65 & 4.81 \\ 0.150 & \\ 0.360 & \end{array}$

